\title{
Fault Estimation and Control for a Quad-rotor MAV using a Polynomial Observer. Part I: Fault Detection
}

\author{
Gerardo R. Flores-Colunga ${ }^{\star}$, H. Aguilar-Sierra ${ }^{\dagger}$, R. Lozano ${ }^{\ddagger}$, and S. Salazar ${ }^{\dagger}$ \\ * is with the Heudiasyc UMR 6599 Laboratory, University of Technology of \\ Compiègne, France. \\ $\dagger$ are with LAFMIA UMI 3175, Cinvestav, México. \\ $\ddagger$ is with the Heudiasyc UMR 6599 Laboratory, UTC CNRS France and LAFMIA \\ UMI 3175, Cinvestav, México. \\ gfloresc@hds .utc.fr, haguilar@ctrl. cinvestav.mx, rlozano@hds . utc.fr, sergio. \\ salazar.cruz@gmail.com \\ http://www.hds.utc.fr
}

\begin{abstract}
This work addresses the problem of fault detection and diagnosis (FDD) for a quad-rotor mini aerial vehicle (MAV). Actuator faults are considered on this paper. The basic idea behind the proposed method is to estimate the faults signals using the extended state observers theory. To estimate the faults, a polynomial observer is presented by using the available measurements and know inputs of the system. In order to investigate the observability and diagnosability properties of the system, a differential algebra approach is proposed. Furthermore, an evaluation function depending on the system states is developed, in order to be used in a controller, which will compensate the failures. The effectiveness of the methodology is illustrated by means of numerical simulations and some experimental tests.
\end{abstract}

Keywords: Quad-rotor, polynomial observer, diagnosability, fault detection and diagnosis.

\section{Introduction}

The growing development in research on MAVs and the consequent improvement of technologies like microcomputers, vision systems and other sensor devices, have increased the performance requirements of such kind of systems. Problems related to trajectory tracking, flight-formation, vision-based localization and lately MAV equipped with manipulators, have been widely researched in the last few years. Therefore, a good performance in the inner-loop of such flight envelopes is needed.

\footnotetext{
* This work was partially supported by the Institute for Science \& Technology of Mexico City (ICyTDF) and the Mexican National Council for Science and Technology (CONACYT).
} 
A wide range of nonlinear control techniques like backstepping [1], [2], singular perturbation techniques [3], sliding modes and switching control [4], [5], have been treated to deal with the complex dynamics of the quad-rotor.

Due to the high cost of the MAV equipment, it is imperative to provide such systems with a fault-control loop, responsible for the identification of possible faults presented at any time of the flight envelope.

Motivated by the fault diagnosis problem, which is the problem of observing fault signals, and the necessity of developing sufficiently robust controllers to cope the presence of likely faults, this research work deals not only with the MAV stabilization problem, but also with the identification of actuator faults. Few works dealing with the fault diagnosis problem applied on quad-rotors are presented in the literature [6], [7], [8], [9], [10].

Taking the attitude, position, angular and translational velocities of the quadrotor MAV as available measurements, we develop a solution for the fault diagnosis problem by means of the differential algebraic approach. With this approach, it is possible to construct a bank of observers in order to implement a scheme of residual generation for fault diagnosis [11], or implement a control law based on state estimation [12]. Thus, it is possible to combine different schemes of nonlinear observers. In [13], the authors present a reduced order and a sliding mode observer, to reconstruct faults in an experimental task, for the case when only one output is available. A reduced order observer and an algebraic observer is presented in [14]. The approach given in [15] is used for fault detection and fault estimation of a wound-rotor induction motor (WRIM). In [16] a polynomial observer, a reduced order observer and a sliding mode observer are used in order to estimate an reconstruct the system states and faults for the case of multiple available outputs. In [17], the polynomial observer is used for the synchronization of chaotic systems.

The paper is organized as follows. The fault diagnosis problem is formulated in Section 2. In order to estimate not only the system states but also the faults dynamics, an extended Luenberger observer called polynomial observer is developed in Section 3. Next, in Section 4, the results previously obtained is applied to the Quad-rotor MAV. Section 6 presents some simulation results for the fault reconstruction problem. Finally, some conclusions and future works are presented in section 7 .

\section{Fault Diagnosis Problem}

The Fault Detection and Diagnosis (FDD) task has the goal of detecting the presence of a fault and construct an estimate of the unknown fault dynamics. Such faults can affect directly the performance of the system components. Therefore, a FDD scheme provides all the necessary information about faults, such as presence (time), type (actuator/sensor) and dynamics (magnitude and form). Thus, based on this information, it is possible to design a system reconfiguration to minimize the fault effects. We begin by defining the terms fault and failure as follows: 
Fault: An undesired change in a system parameter or variable that reduces the performance/magnitude of one component of its nominal value. In summary, a fault is an unacceptable tolerable malfunction.

Failure: A complete breakdown of the system, caused by a catastrophic malfunction of one or more components of the system. In summary, a failure is an intolerable malfunction.

Throughout this work, we describe a class of nonlinear systems with faults as follows

$$
\begin{aligned}
& \dot{x}(t)=g(x, u, f) \\
& y(t)=h(x, u)
\end{aligned}
$$

where

$x \in \mathbb{R}^{n}$ is the state vector

$u \in \mathbb{R}^{m}$ is the vector of known inputs

$f \in \mathbb{R}^{\mu}$ is the faults vector (unknown inputs)

$y \in \mathbb{R}^{p}$ is the outputs vector

In this paper, we consider only the case of faults in the actuators. So, we introduce the concept of observability and diagnosability in the field of the differential algebra.

\subsection{Observability and Diagnosability Condition}

The observability and diagnosability notion of a system, linear or nonlinear in the differential algebra approach need a basic definition. Further details can be found in [13].

Definition 1. For the system described by (1) a state $x$ is said to be observable if it is possible to estimate the state by means of the available measurements of the system, so we say that $x$ is observable if it is algebraically observable, i.e., the state $x$ satisfies a polynomial equation in terms of $u$ and $y$ and some of their time derivatives:

$$
P\left(x, y, \dot{y}, \ddot{y}, \ldots, y^{(n)}, u, \dot{u}, \ddot{u}, \ldots, u^{(n)}\right)=0
$$

Definition 2. A fault $f$ is said to be diagnosable if it is possible to estimate the fault from the available measurements of the system, i.e., $f$ is diagnosable if it is algebraically observable if it satisfies a polynomial equation in terms of $u$ and $y$ and some of their time derivatives:

$$
P\left(f, y, \dot{y}, \ddot{y}, \ldots, y^{(n)}, u, \dot{u}, \ddot{u}, \ldots, u^{(n)}\right)=0
$$

Remark 1. The diagnosability condition is independent of the observability of the system.

Referring to system (1), the vector $f$ contains the unknown inputs. In order to estimate its uncertain dynamics, the state vector is extended to deal with the 
fault vector. So, we can rewritten the system in an extended form as follows

$$
\left\{\begin{aligned}
\dot{x}(t) & =g(x, u, f) \\
\dot{f}_{k}(t) & =\Omega_{k}(x, u, f) \quad 1 \leq k \leq \mu \\
y(t) & =h(x, u)
\end{aligned}\right.
$$

The following results from the theory of differential algebraic are on useful tool to determine whether a fault can be reconstructed from the know inputs and available outputs.

Theorem 1. Assume that the system (1) is diagnosable, then the number of faults is less or equal to the number of available measurements (outputs), i.e.

$$
\mu \leq p
$$

The proof of Theorem 1 can be seen in [18].

\section{Polynomial Observer}

The polynomial observer, is a scheme that combines two kinds of observers. The first one is like an extended Luenberger observer which is used to reconstructing and estimate the system states. While the second one is a free mode observer, which has the function of reconstruct an estimate of the faults dynamics. The polynomial observer can be seen as a Taylor series, where the first-order term is the observed state, thus improving the performance and speed of convergence including terms of high-order correction in the structure. It is worth mentioning that this scheme is considered for the case of multiple outputs available, where the terms of higher order correction are odd powers and are a linear combination of the observation errors of each output available and the order of the polynomial compensations is a determining factor for the parameter " $q$ ".

Consider the system with presence of faults, given in (4), the observation problem for the unknown vector of faults can be estimated using a polynomial observer. Therefore the system (4) can be rewritten as

$$
\left\{\begin{array}{rl}
\dot{x}(t) & =A x+\Psi(x, \bar{u}) \\
\dot{f}_{k}(t) & =\Omega_{k}(x, \bar{u}) \\
y(t) & =C x
\end{array} \quad 1 \leq k \leq \mu\right.
$$

where $\left\|\Omega_{k}(x, \bar{u})\right\| \leq N, N \in \mathbb{R}^{+}$and $\Psi(x, \bar{u})$ is a nonlinear function that satisfies the Lipschitz condition, with $\bar{u}=(u, f)$ uniformly bounded.

$$
\|\Psi(x, \bar{u})-\Psi(\hat{x}, \bar{u})\| \leq L\|x-\hat{x}\|
$$

\subsection{Observer design}

Now, consider the system with faults (5), the following lemma describes the construction of the polynomial observer. 
Lemma 1. Let the system (5) be algebraically diagnosable, then, the following nonlinear system is a full order state observer for the given system

$$
\left\{\begin{aligned}
\dot{\hat{x}}(t)= & A \hat{x}+\Psi(\hat{x}, u, \hat{f})+ \\
& +\sum_{i=1}^{p} \sum_{j=1}^{q} K_{i j}\left(y_{i}-C_{i} \hat{x}\right)^{2 j-1} \\
\dot{\hat{f}}_{k}(t)= & \sum_{l=1}^{q} \bar{K}_{k l}\left(f_{k}-\hat{f}_{k}\right)^{2 l-1}
\end{aligned}\right.
$$

Where

$A \in \mathbb{R}^{n \times n}$

$\hat{x} \in \mathbb{R}^{n \times 1}$ is the estimate of the state $x$

$\hat{f}_{k} \in \mathbb{R}^{\mu}$ is the estimate of faults vector $f$

$q \in \mathbb{R}^{+}$

$\Psi(\hat{x}, u, \hat{k}) \in \mathbb{R}^{n \times 1}$

$\left[K_{i j}\right]_{\substack{1 \leq i \leq p \\ 1 \leq j \leq q}},\left[\bar{K}_{k l}\right]_{\substack{1 \leq k \leq \mu \\ 1 \leq l \leq q}}$ are positive gains

where $\hat{x}_{0}=\hat{x}\left(t_{0}\right)$ and $\hat{f}_{k 0}=\hat{f}_{k}\left(t_{0}\right)$ are arbitrary initial conditions, the parameter $q$ determines the order of the polynomial compensation. To ensure the observer convergence, the following assumptions are considered:

A1: $f_{k}(t)$ is algebraically observable

A2: The gains $\left[K_{i l}\right]_{1 \leq i \leq p}$ can be chosen such that the following algebraic Riccati equation has a symmetric and positive definite solution $P$ for some $\epsilon>0$

$$
\left(A-\sum_{i=1}^{p} K_{i 1} C_{i}\right)^{T} P+P\left(A-\sum_{i=1}^{p} K_{i 1} C_{i}\right)+L^{2} P P+I+\epsilon I=0
$$

A3: The gains $\left[K_{i j}\right]_{\substack{1 \leq i \leq p \\ 2 \leq j \leq q}}$ are chosen such that

$$
\lambda_{\text {min }}\left(\left(P K_{i j} C_{i}\right)^{T}+\left(P K_{i j} C_{i}\right)\right) \geq 0
$$

We define the estimation error vector as $e=\left[e_{x}, e_{k}\right]^{T}$, whit $e_{x}=x-\hat{x}$ and $e_{k}=f_{k}-\hat{f}_{k}$. So from the systems (5) and (7), we determine the dynamics for the corresponding error estimation

$$
\begin{aligned}
\dot{e}_{x}= & \left(A-\sum_{i=1}^{p} K_{i 1} C_{i}\right) e_{x}- \\
& -\sum_{i=1}^{p} \sum_{j=2}^{q} K_{i j}\left(C_{i} e_{x}\right)^{2 j-1}+ \\
& +[\Psi(x, \bar{u})-\Psi(\hat{x}, \bar{u})] \\
\dot{e}_{k}= & \Omega_{k}-\bar{K}_{k 1} e_{k}-\sum_{j=2}^{q} \bar{K}_{k j}\left(e_{k}\right)^{2 l-1}
\end{aligned}
$$




\subsection{Convergence Analysis}

In order to ensure the convergence to zero of the estimation error, we establish the following theorem.

Theorem 2. For the system (5), suppose that $x(t) \exists \forall t \geq 0$, the function $\Psi(x, \bar{u})$ satisfies the Lipschitz condition given in $(6)$, and $x(t), f(t)$ are algebraically observable. Thus, if there exists a positive definite matrix $P$ and positive observer gains $K_{i j}, \bar{K}_{k l}$ such that the system (7) is an observer for system (5), then the estimation error converges to zero asymptotically.

Proof. Consider the following Lyapunov function candidate

$$
\begin{aligned}
& V=V_{1}+V_{2} \\
& V_{1}=e_{x}^{T} P e_{x} ; V_{2}=\frac{1}{2} e_{k}^{2}
\end{aligned}
$$

where the matrix $P$ satisfies the assumption A2.

The proof of theorem 2, is developed in two parts as follows:

i) The time derivative of $V_{1}$ is given as

$$
\begin{aligned}
\dot{V}_{1} & =\dot{e}_{x}^{T} P e_{x}+e_{x}^{T} P \dot{e}_{x} \\
& =e_{x}^{T}\left(\left(A-\sum_{i=1}^{p} K_{i 1} C_{i}\right)^{T} P+P\left(A-\sum_{i=1}^{p} K_{i 1} C_{i}\right)\right) e_{x}+ \\
& +2 e x^{T} P[\Psi(x, \bar{u})-\Psi(\hat{x}, \bar{u})]- \\
& -2 \sum_{i=1}^{p} \sum_{j=2}^{q} K_{i j}\left(C_{i} e_{x}\right)^{2 j-2} e_{x}^{T}\left(\left(P K_{i j} C_{i}\right)^{T}+\left(P K_{i j} C_{i}\right)\right) e_{x}
\end{aligned}
$$

From the follow inequality based on the Lipschitz condition

$$
2 e x^{T} P[\Psi(x, \bar{u})-\Psi(\hat{x}, \bar{u})] \leq L^{2} e_{x}^{T} P P e_{x}+e_{x}^{T} e_{x}
$$

and using the Rayleigh's inequality together with assumption A3. it follows that

$$
-e_{x}^{T} P K_{i j} C_{i} e_{x} \leq-\lambda_{\min }\left(P K_{i j} C_{i}+\left(P K_{i j} C_{i}\right)^{T}\right)\left\|e_{x}\right\|^{2}
$$

Therefore, applying inequalities (10) and (11) we have

$$
\begin{aligned}
\dot{V}_{1} \leq & e_{x}^{T}\left[\left(A-\sum_{i=1}^{p} K_{i 1} C_{i}\right)^{T} P+P\left(A-\sum_{i=1}^{p} K_{i 1} C_{i}\right)+\right. \\
& \left.+L^{2} P P+I\right] e_{x}- \\
& -2 \sum_{i=j}^{p} \sum_{j=2}^{q} K_{i j}\left(C_{i} e_{x}\right)^{2 j-2} \lambda_{\min }\left(P K_{i j C_{i}}+\left(P K_{i j C_{i}}\right)^{T}\right)\left\|e_{x}\right\|^{2} \\
& \leq e_{x}^{T}\left[\left(A-\sum_{i=1}^{p} K_{i 1} C_{i}\right)^{T} P+P\left(A-\sum_{i=1}^{p} K_{i 1} C_{i}\right)+\right. \\
& \left.+L^{2} P P+I\right] e_{x} \\
& =-\epsilon\left\|e_{x}\right\|^{2}
\end{aligned}
$$

ii) In the same way, for the second term in the Lyapunov function candidate, we obtain the time derivative of $V_{2}$ as

$$
\begin{aligned}
\dot{V}_{2} & =e_{k} \dot{e}_{k} \\
& =e_{k}\left(\Omega_{k}-\bar{K}_{k 1} e_{k}-\sum_{l=2}^{q} \bar{K}_{k l} e_{k}^{2 l-1}\right) \\
& =e_{k} \Omega_{k}-\bar{K}_{k 1} e_{k}^{2}-\sum_{l=2}^{q} \bar{K}_{k l} e_{k}^{2 l} \\
& \leq\left|e_{k}\right|\left|\Omega_{k}\right|-\bar{K}_{k 1} e_{k}^{2} \\
& \leq\left|e_{k}\right| N-\bar{K}_{k 1}\left|e_{k}\right|^{2} \\
& =-\left[\bar{K}_{k 1}\left|e_{k}\right|-N\right]\left|e_{k}\right|
\end{aligned}
$$


$\dot{V}_{2}$ is negative inside the set $\left\{\left|e_{k}\right|>N / \bar{K}_{k 1}\right\}$, i.e., exists $\bar{\epsilon}>0$ such that $\bar{K}_{k 1}\left|e_{k}\right|-N=\bar{\epsilon}>0$.

We prove that $\left|e_{k}\right|$ is upper bounded. Now let constants $\alpha, \beta$ upper bounds of $V_{2}\left(e_{k}\right)$. With $\beta>\frac{N^{2}}{2 K_{k 1}^{2}}$, the solution that initiates in the set $\left\{V_{2}\left(e_{k}\right) \leq \beta\right\}$ will remain inside that set for all $t \geq 0$, because $\dot{V}_{2}$ is negative in $V_{2}=$ $\beta$. Therefore the solution of $\dot{e}_{k}$ is uniformly bounded [19]. Furthermore, if $\frac{N^{2}}{2 K_{k 1}^{2}}<\alpha<\beta$, then $\dot{V}_{2}$ will be negative in the set $\left\{\alpha \leq V_{2} \leq \beta\right\}$. In this set $V_{2}$ will decrease monotonously until the solution is in the set $\left\{V_{2} \leq \alpha\right\}$. According to [19] the solution is uniformly ultimately bounded with ultimate bound $\left|e_{k}\right| \leq \sqrt{2 \alpha}$. For example, if we define $\alpha=\frac{N^{2}}{2 K_{k 1}^{2}}$ and $\beta=\frac{N^{2}}{K_{k 1}^{2}}$, the ultimate bound is

$$
\left|e_{k}\right| \leq \frac{N}{\bar{K}_{k 1}}
$$

Hence

$$
\dot{V}_{2} \leq-\bar{\epsilon}\left|e_{k}\right|
$$

Finally, from (i) and (ii), we conclude that

$$
\dot{V} \leq-\epsilon\left\|e_{x}\right\|^{2}-\bar{\epsilon}\left|e_{k}\right|<0
$$

\section{Application to Quad-rotor MAV}

In this section, the polynomial observer approach developed in Section 3 is applied to a Quad-rotor MAV. We will state the mathematical model of the MAV and some notations. Then, the diagnosability analysis of such vehicle dynamics is developed.

\subsection{Modeling}

The Quad-rotor mathematical model using the corresponding coordinate system shown in Figure (1) is given as follows

$$
\begin{aligned}
m \ddot{x} & =\left(u_{1}+u_{2}+u_{3}+u_{4}\right)\left(S_{\psi} S_{\phi}+C_{\psi} S_{\theta} C_{\phi}\right) \\
m \ddot{y} & =\left(u_{1}+u_{2}+u_{3}+u_{4}\right)\left(S_{\psi} S_{\theta} C_{\phi}-C_{\psi} S_{\phi}\right) \\
m \ddot{z} & =m g-\left(u_{1}+u_{2}+u_{3}+u_{4}\right)\left(C_{\phi} C_{\theta}\right) \\
\ddot{\theta} & =u_{3}-u_{1} \\
\ddot{\phi} & =u_{2}-u_{4} \\
\ddot{\psi} & =u_{1}-u_{2}+u_{3}-u_{4}
\end{aligned}
$$

where $u_{i}$ are the control inputs, $(x, y, z)$ the position states and $(\theta, \phi, \psi)$ the orientation angles. Using the model (12), the following changes of coordinates is made in order to formulate the problem

$$
\begin{array}{rrrrrr}
x_{1}=x & x_{3}=y & x_{5}=z & x_{7}=\theta & x_{9}=\phi & x_{11}=\psi \\
x_{2}=\dot{x} & x_{4}=\dot{y} & x_{6}=\dot{z} & x_{8}=\dot{\theta} & x_{10}=\dot{\phi} & x_{12}=\dot{\psi}
\end{array}
$$




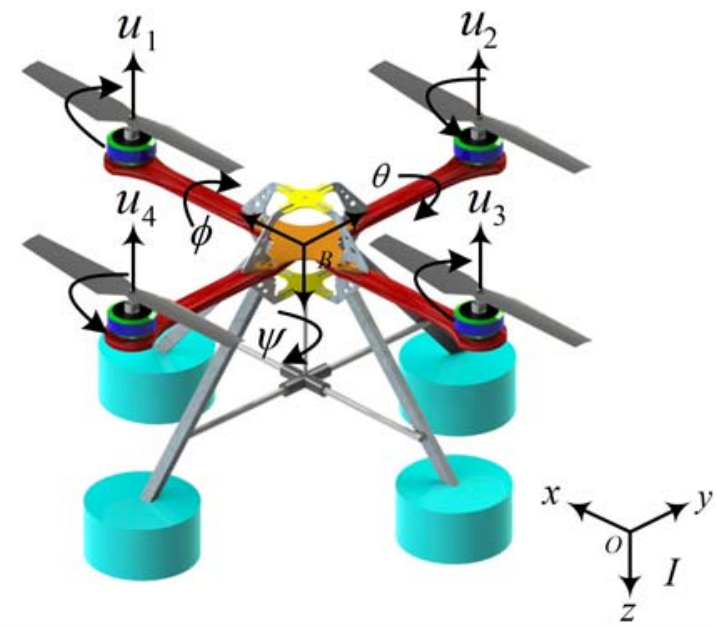

Fig. 1. The three-dimensional quad-rotor model.

With this notation, the state vector is given by $x=\left[x_{1}, x_{2}, \ldots, x_{12}\right]^{T}$ and the input vector as $u=\left[u_{1}, u_{2}, u_{3}, u_{4}\right]$. For a Quad-rotor MAV, we consider a fault as a reduction of the performance of one or two actuator, with the constraint that if the failure affects an actuator, the second failure will affect the opposite actuator by the symmetry of the structure, i.e. the failure effects can only be minimized when these occurs in the following form:

1. The fault effects only one actuator

2. The fault affects the pairs $\left(u_{1}, u_{3}\right)$ or $\left(u_{2}, u_{4}\right)$

Other wise we would have a catastrophic malfunction and it would be impossible to minimize the fault effects.

We now consider the presents of a fault on four of the engines, so we define the input with presents of fault as $\bar{u}_{k}=u_{k}+f_{k}$. Therefore the system with faults is given by:

$$
\begin{aligned}
\dot{x}_{1} & =x_{2} \\
\dot{x}_{2} & =\frac{1}{m}\left(\bar{u}_{1}+\bar{u}_{2}+\bar{u}_{3}+\bar{u}_{4}\right)\left(S_{x_{11}} S_{x_{9}}+C_{x_{11}} S_{x_{7}} C_{x_{9}}\right) \\
\dot{x}_{3} & =x_{4} \\
\dot{x}_{4} & =\frac{1}{m}\left(\bar{u}_{1}+\bar{u}_{2}+\bar{u}_{3}+\bar{u}_{4}\right)\left(S_{x_{11}} S_{x_{7}} C_{x_{9}}-C_{x_{11}} S_{x_{9}}\right) \\
\dot{x}_{5} & =x_{6} \\
\dot{x}_{6} & =g-\frac{1}{m}\left(\bar{u}_{1}+\bar{u}_{2}+\bar{u}_{3}+\bar{u}_{4}\right)\left(C_{x_{9}} C_{x_{7}}\right) \\
\dot{x}_{7} & =x_{8} \\
\dot{x}_{8} & =\bar{u}_{3}-\bar{u}_{1} \\
\dot{x}_{9} & =x_{10} \\
\dot{x}_{10} & =\bar{u}_{2}-\bar{u}_{4} \\
\dot{x}_{11} & =x_{12} \\
\dot{x}_{12} & =\bar{u}_{1}-\bar{u}_{2}+\bar{u}_{3}-\bar{u}_{4}
\end{aligned}
$$


where $f_{k}$ are additive faults that which affect directly the performance of the engines that produce the thrust inputs $u_{k}$.

\subsection{Control Strategy}

The proposed control strategy is based on the idea that the global system (12) is constituted of two subsystems, the attitude dynamics and the position dynamics, each one with a time-scale separation between them [20]. From this fact, it is possible to propose a hierarchical control scheme where the position controller provides desired attitude angles $\phi_{d}, \theta_{d}$ which are the angles to be tracked by the orientation controllers. We have implemented a nonlinear control strategy based on this principle. In this paper we don't present the control strategy, due this is not the purpose of the present work, however the interested lector can see more details about the control strategy applied on this paper in [3].

\subsection{Diagnosability analysis}

From Theorem 1 it is required that the number of faults $(\mu=4)$ be less or equal to available measurements. For this case, we consider the output vector as $y=\left[y_{1}, y_{3}, y_{5}, y_{7}, y_{9}, y_{11}\right]=\left[x_{1}, x_{3}, x_{5}, x_{7}, x_{9}, x_{11}\right]$. i Taking into account the above mentioned considerations, the condition from theorem 1 is hold with $4=$ $\mu<p=6$. To determine the diagnosability of the system (13), we evaluate the algebraic diagnosability condition given in definition 2 . For the considered faults, inputs and outputs, the system (13) results in

$$
\begin{aligned}
\ddot{y_{2}} & =\frac{1}{m}\left(\bar{u}_{1}+\bar{u}_{2}+\bar{u}_{3}+\bar{u}_{4}\right)\left(S_{y_{11}} S_{y_{9}}+C_{y_{11}} S_{y_{7}} C_{y_{9}}\right) \\
\ddot{y_{3}} & =\frac{1}{m}\left(\bar{u}_{1}+\bar{u}_{2}+\bar{u}_{3}+\bar{u}_{4}\right)\left(S_{y_{11}} S_{y_{7}} C_{y_{9}}-C_{y_{11}} S_{y_{9}}\right) \\
\ddot{y_{5}} & =g-\frac{1}{m}\left(\bar{u}_{1}+\bar{u}_{2}+\bar{u}_{3}+\bar{u}_{4}\right)\left(C_{y_{9}} C_{y_{7}}\right) \\
\ddot{y_{7}} & =\bar{u}_{3}-\bar{u}_{1} \\
\ddot{y_{9}} & =\bar{u}_{2}-\bar{u}_{4} \\
\ddot{y_{11}} & =\bar{u}_{1}-\bar{u}_{2}+\bar{u}_{3}-\bar{u}_{4}
\end{aligned}
$$

From system (14), we have that

$$
\begin{aligned}
\frac{m\left(g-\ddot{y}_{6}\right)}{C_{y_{9}} C_{y_{7}}} & =u_{1}+f_{1}+u_{2}+f_{2}+u_{3}+f_{3}+u_{4}+f_{4} \\
\ddot{y}_{7} & =u_{3}+f_{3}-u_{1}-f_{1} \\
\ddot{y}_{9} & =u_{2}+f_{2}-u_{4}-f_{4} \\
\ddot{y}_{11} & =u_{1}+f_{1}-u_{2}-f_{2}+u_{3}+f_{3}-u_{4}-f_{4}
\end{aligned}
$$

Adding (15a) and (15d)

$$
\frac{m\left(g-\ddot{y}_{5}\right)}{C_{y_{9}} C_{y_{7}}}+\ddot{y}_{11}=u_{1}+f_{1}+2 u_{3}+2 f_{3}
$$

Adding 2(15b) and (16)

$$
f_{3}=\frac{m\left(g-\ddot{y}_{5}\right)}{4 C_{y_{9}} C_{y_{7}}}+\frac{1}{2} \ddot{y}_{7}+\frac{1}{4} \ddot{y}_{11}-u_{3}
$$


Replacing (17) into (15b)

$$
f_{1}=\frac{m\left(g-\ddot{y}_{5}\right)}{4 C_{y_{9}} C_{y_{7}}}-\frac{1}{2} \ddot{y}_{7}+\frac{1}{4} \ddot{y}_{11}-u_{1}
$$

Adding, (15a) and (15c)

$$
\frac{m\left(g-\ddot{y}_{5}\right)}{C_{y_{9}} C_{y_{7}}}+\ddot{y}_{9}=u_{1}+f_{1}+2 u_{2}+2 f_{2}+u_{3}+f_{3}
$$

Replacing (18) and (17) into (19)

$$
f_{2}=\frac{m\left(g-\ddot{y}_{5}\right)}{4 C_{y_{9}} C_{y_{7}}}+\frac{1}{2} \ddot{y}_{9}+\frac{1}{4} \ddot{y}_{11}-u_{2}
$$

Finally, replacing (20) into (15c) it follows that

$$
f_{4}=\frac{m\left(g-\ddot{y}_{5}\right)}{4 C_{y_{9}} C_{y_{7}}}-\frac{1}{2} \ddot{y}_{9}+\frac{1}{4} \ddot{y}_{11}-u_{4}
$$

Therefore, from equations (17), (18), (20) and (21) we conclude that the system (13) is diagnosable, with the considered inputs and outputs.

\subsection{Polynomial Observer}

The system (13), can be expressed in a similar way as in (5) with:

$A \in \mathbb{R}^{12 \times 12}$ where the elements of the matrix are given as follows: $a_{1,2}=$ $a_{3,4}=a_{5,6}=a_{7,8}=a_{9,10}=a_{11,12}=1$ and zero other wise.

The nonlinear function $\Psi(x, \bar{u})=\left[\psi_{1}, p s i_{2}, \ldots, \psi_{12}\right]$ is given by:

$$
\begin{aligned}
& \psi_{1}=\psi_{=}=\psi_{5}=\psi_{7}=\psi_{9}=\psi_{11}=0 \\
& \psi_{2}=\frac{1}{m}\left(\bar{u}_{1}+\bar{u}_{2}+\bar{u}_{3}+\bar{u}_{4}\right)\left(S_{x_{11}} S_{x_{9}}+C_{x_{11}} S_{x_{7}} C_{x_{9}}\right) \\
& \psi_{4}=\frac{1}{m}\left(\bar{u}_{1}+\bar{u}_{2}+\bar{u}_{3}+\bar{u}_{4}\right)\left(S_{x_{11}} S_{x_{7}} C_{x_{9}}-C_{x_{11}} S_{x_{9}}\right) \\
& \psi_{6} g=-\frac{1}{m}\left(\bar{u}_{1}+\bar{u}_{2}+\bar{u}_{3}+\bar{u}_{4}\right)\left(C_{x_{11}} C_{x_{9}}\right) \\
& \psi_{8}=\bar{u}_{3}-\bar{u}_{1} \\
& \psi_{10}=\bar{u}_{2}-\bar{u}_{4} \\
& \psi_{12}=\bar{u}_{1}-\bar{u}_{2}+\bar{u}_{3}-\bar{u}_{4}
\end{aligned}
$$

So, the following system is a polynomial observer for the given system

$$
\left\{\begin{aligned}
\dot{\hat{x}}(t)= & A \hat{x}+\Psi(\hat{x}, u, \hat{f})+ \\
& +\sum_{i=1}^{6} \sum_{j=1}^{3} K_{i j}\left(y_{i}-C_{i} \hat{x}\right)^{2 j-1} \\
\dot{\hat{f}}_{k}(t)= & \sum_{l=1}^{3} \bar{K}_{k l}\left(f_{k}-\hat{f}_{k}\right)^{2 l-1}
\end{aligned}\right.
$$

Where we fixed the parameter $q=3$ and the $f_{1 \leq k \leq 4}$ are given by (18), (20), (17), (21). 


\section{Evaluation Function}

In this section, an evaluation function is presented in order to use the estimate of the failure with the methodology developed in the previous section. Such evaluation function is presented in a way that could be used in a controller to compensate the failure.

Without loss of generality, we present the evaluation function for the roll dynamics, but the same procedure could be developed for the pitch and yaw dynamics.

\subsection{Fault detection}

In order to detect the fault and at the same time differentiate it from perturbations inherent in the system, we define the evaluation function $e_{f}$ as a function of system states as follows

$$
e_{f}=\frac{1}{\left(1+e^{-c_{b}(\phi-b)}\right)\left(1+e^{-c_{a}(\dot{\phi}-a)}\right)}
$$

where parameters $c_{a}$ and $c_{b}$ are positive real numbers which define the fault slope for $\phi$ and $\dot{\phi}$ respectively. The parameters $a$ and $b$ represent the position and velocity in roll dynamics for which there will be a fault. Accordingly, they are the boundaries between a disturbance and a fault.

So, the basic idea is to estimate the disturbances and then use them in the evaluation function (23). In this way, one should predefine the parameter values $a, b$. So, by means of an observation of the system dynamics, one knows the existence of a fault. This approach allows to determine the existence of a fault without any measurement of engine speed, eliminating the use of additional sensors on the platform.

In addition, a control strategy could be implemented by using (23), due such evaluation function is continuously differentiable and furthermore, it depends on the system states $(\phi, \dot{\phi})$.

\subsection{Experimental results by using the evaluation function}

In this subsection, some experimental results are presented to visualize the performance of the evaluation function at real-time experiments. The experimental results have been tested on the Quad-rotor experimental platform developed at the HEUDIASYC Laboratory. More details about this platform can be seen in [21]. We have simulated the failures by disturbing the Quad-rotor platform in the roll dynamics. For these tests, we have used the parameters shown in table 1 .

Two motors are involved in the pitch dynamics (Fig. 1). Thus, if one of this motors fails, its velocity will be reduced or augmented, causing an aggressive rolling moment due to the difference of thrust between the faulty motor and the operating motor. Therefore, we can evaluate such moment by inspecting the pitching dynamics, i.e. the behavior of the states $(\theta, \dot{\theta})$. In order to visualize the behavior of the evaluation function on the real-time experiments, we have perturbed the Quad-rotor platform on its roll axis. Fig. 2 shows the performance of the evaluation function (23). As we see in this figure, the disturbances lower than $20 \mathrm{deg}$ have been omitted by the evaluation function, while the disturbances greater or equal to $20 \mathrm{deg}$ have been taken as failures. With this approach, we can tune the parameters given in table 1 , in order to choose a desired performance to tell the system how to distinguish a perturbation of a fault. 
Table 1. The parameters of the Quad-rotor MAV considered for the experimental tests.

\begin{tabular}{c||c}
\hline Parameter & Value \\
\hline \hline$c_{b}$ & 1 \\
$c_{a}$ & 1 \\
$b$ & $20 \mathrm{deg}$ \\
$a$ & $10 \mathrm{deg} / \mathrm{sec}$ \\
\hline
\end{tabular}
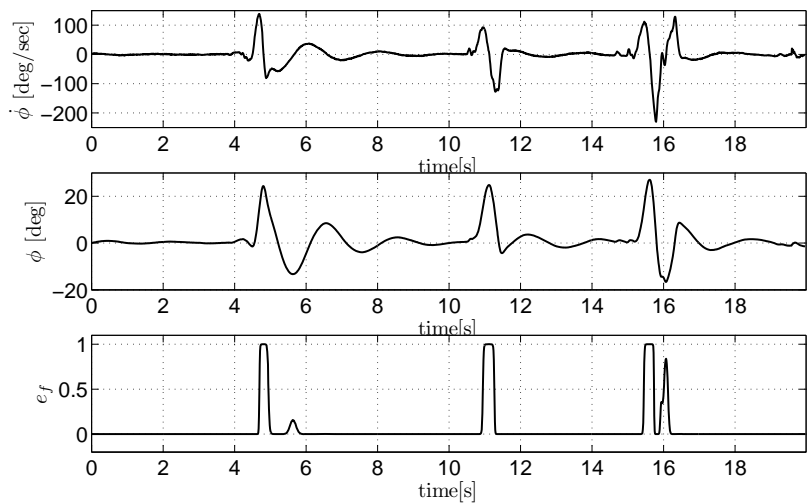

Fig. 2. Evaluation function (23) and states of the roll dynamics.

\section{$6 \quad$ Fault reconstruction results}

In this section, we present some simulation results of the procedure developed in Section 3. The dynamics of the Quad-rotor MAV and the fault dynamics have been simulated using MATLAB Simulink. For the simulation procedure, we have consider the following conditions: The desired values for the position dynamics are $x_{d}=y_{d}=0 \mathrm{~m}$ and $z_{d}=0.75 \mathrm{~m}$ and for the attitude dynamics are $\theta_{d}=\phi_{d}=0$ and $\psi_{d}=45$ degrees. The objective is that the Quad-rotor take off and reaches the desired height and remain stable in that position, in other words, we want that the desired values for the linear and angular velocities are equal to zero. To simplify the calculations we assume that $m=g=1$. A simulation time of $300 \mathrm{~s}$ and a step of $0.001 \mathrm{~s}$ has been chosen.

For all simulation results we have considered that the fault affects the performance of each engine, i.e. the actuators for the Quad-rotor MAV. Four faults were artificially generated as follows

$$
\begin{aligned}
f_{1}= & 0.226\left(1+\sin \left(0.5 t e^{-0.1 t}\right)\right) \mathcal{U}(t-50) \\
f_{2}= & 0.045\left(1+\sin \left(0.076 e^{(t-0.3)}\right)\right) \mathcal{U}(t-20) \\
f_{3}= & 0.055 e^{-0.01(t-0.3)} \mathcal{U}(t-10)+ \\
& +0.068 e^{-0.005(t-1)} \mathcal{U}(t-80)+ \\
& +0.159 e^{-0.07(t-1.3)} \mathcal{U}(t-140) \\
f_{4} & =0.718 e^{-0.01(t-2)} \mathcal{U}(t-30)
\end{aligned}
$$


where $\mathcal{U}(t)$ is the unit step function. The magnitude of the faults were selected very close to the magnitude of the generated thrusts inputs for the case without faults, to obtain better results.

The results of the implementation of the polynomial observer proposed in (22) for the fault diagnosis task, for the considered available outputs and inputs. As shown in the Figure 3, for the fault $f_{1}$. The gain values for the proposed observer were $K_{11}=2.5, K_{12}=34$ and $K_{13}=66$. In the same way in figures 4 , 5 and 6 , we show the estimation result for the faults $f_{2}, f_{3}$ and $f_{4}$, where the gain values for each observer are $K_{21}=5.05, K_{22}=2, K_{23}=1.6, K_{31}=1.5, K_{32}=$ $27, K_{33}=56$ and $K_{41}=4.23, K_{42}=7, K_{43}=3$ respectively.

In order to evaluate the effectiveness of the proposed polynomial observer, we use initial conditions different from zero, to see how long it takes to converge to the actual value of the fault. The initial conditions were $f_{1, c . i}=0.087, f_{2, c . i}=$ $0.065, f_{3, c . i}=0.055$ and $f_{4, c . i}=0.073$.

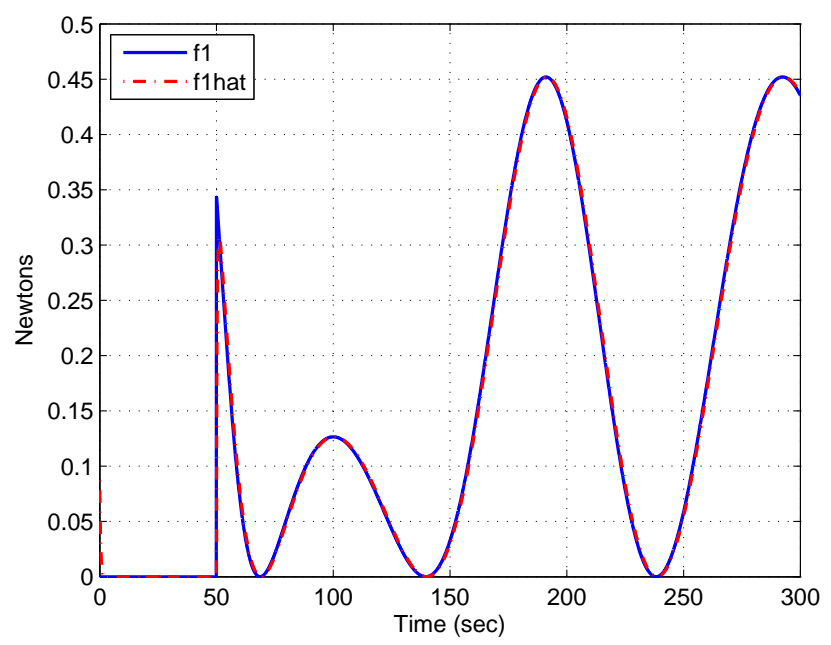

Fig. 3. Estimation result for the fault $f_{1}$

For all faults we obtained good estimation results. As can be seen, the proposed observer converge quickly to the actual values of the faults, and although the approach only considers the case of fault with differentiable dynamics, it is noted that the approach has the capacity to reconstruct abrupt faults as shown in figures 5 and 6 .

The attitude dynamic under the effect of the faults is shown in figure 7 . The direct consequence of the presence of faults on actuators, is that, the controller tries to stabilize the system and bring the dynamics of roll, pitch and yaw to the desired values. However due to thrust limitation, the objective is not fully accomplished .Noticed that the faults affects more the dynamics in yaw.

Figure 8, shows the corresponding angular velocities in roll, pitch and yaw for the case of presence of faults. As can be seen, between in the first 100 seconds the angular velocities change abruptly, because in this time interval, all the faults appear. 


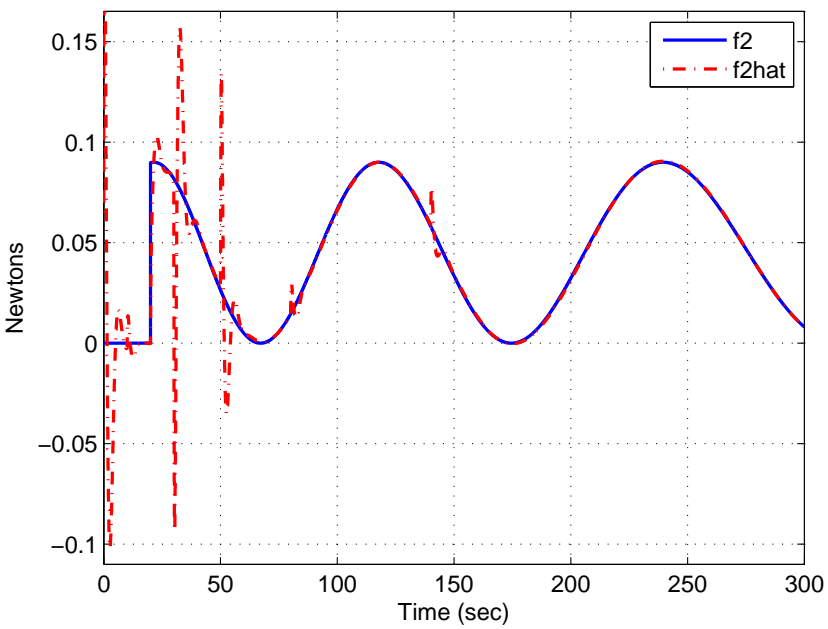

Fig. 4. Estimation result for the fault $f_{2}$

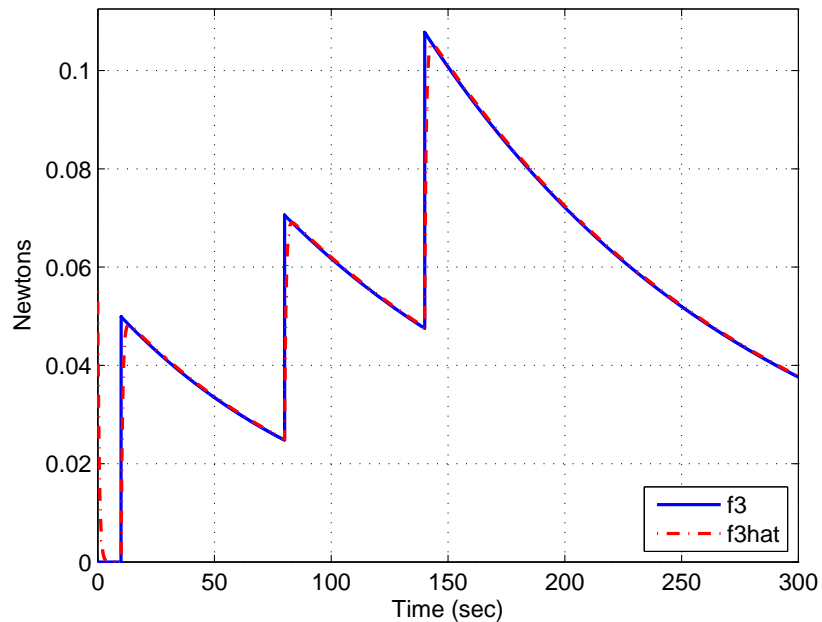

Fig. 5. Estimation result for the fault $f_{3}$

The position dynamics affected for the faults are shown in Figure 9. Note that the difference between the dynamic without faults and with fault is very significant, especially for the dynamics in the $y$-axis, which is very large. The Position controller generates large inputs to try reach the desired position values. However due to the faults the errors grow and the controller is unable to compensate such errors. 


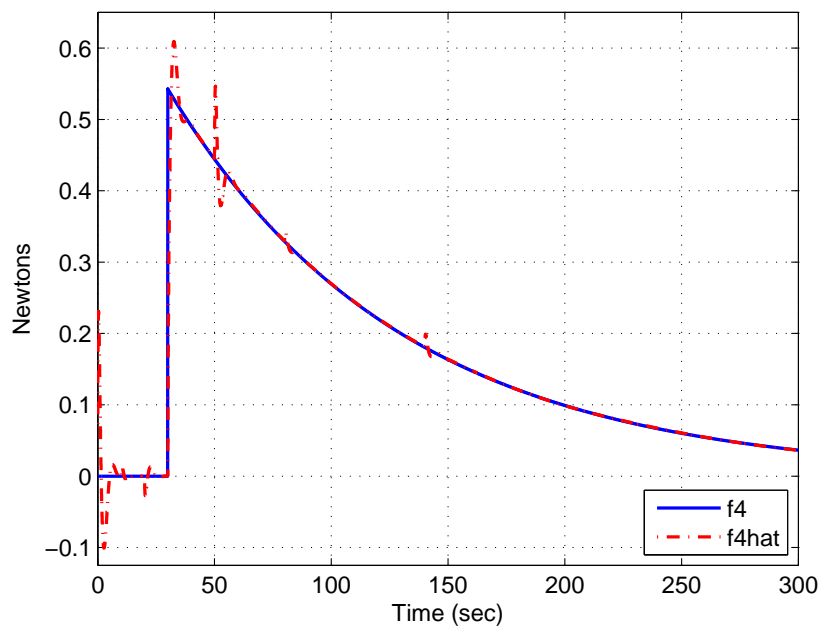

Fig. 6. Estimation result for the fault $f_{4}$
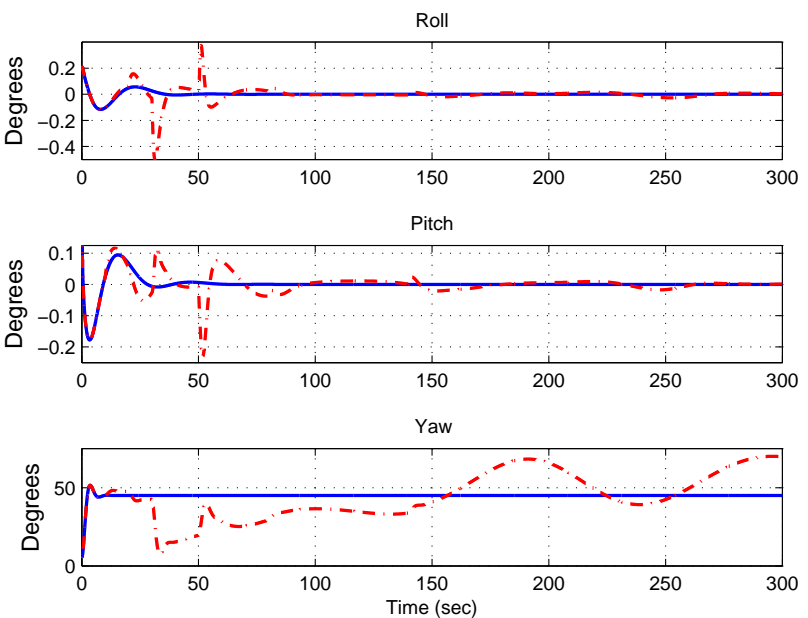

Fig. 7. Comparison for the attitude dynamics for the case without faults (blue line) and with faults (red dash-dot line)

Finally figures 10 and 11 show the control inputs and corresponding thrusts generated by the control strategy. As can be seen in Figure 10 the difference between both cases is very significant, because for the case without faults the control inputs are constant while for the case with faults, the thrusts are nonconstant and larger. The controller tries to compensate the error generated by the presence of the faults, but it shows clearly in Figure 11, where we see that 

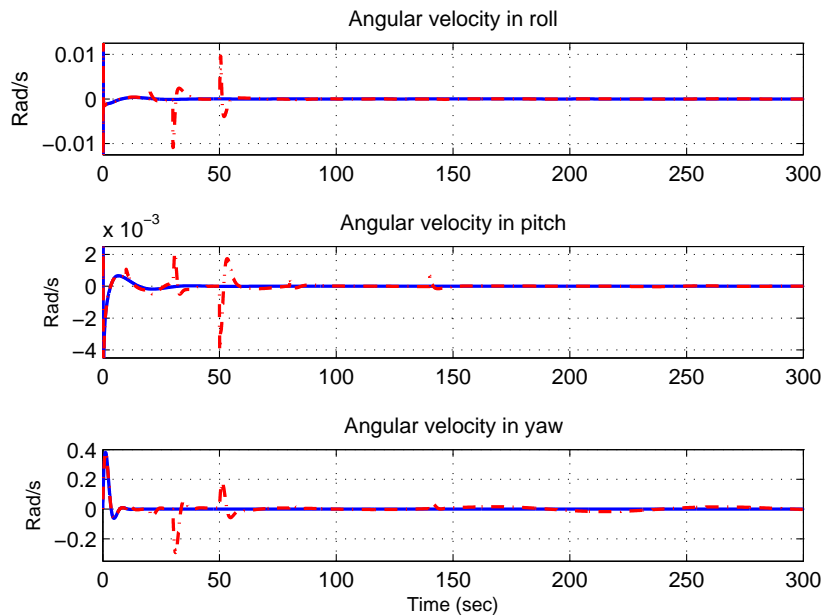

Fig. 8. Comparison for the angular velocity dynamics for the case without faults (blue line) and with faults (red dash-dot line)
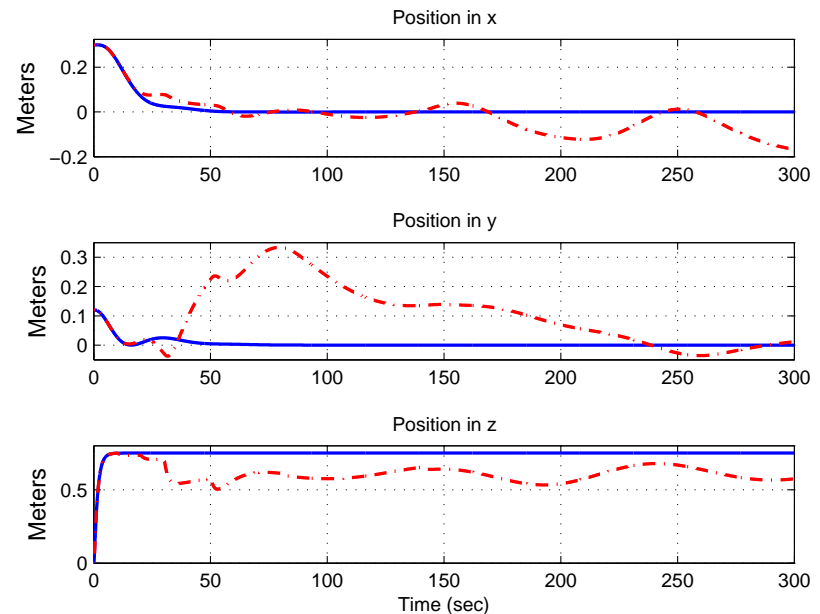

Fig. 9. Comparison for the position dynamics for the case without faults (blue line) and with faults (red dash-dot line)

the corresponding thrust forces inputs are very similar to the dynamics of the faults but with opposite sign. Notice that the faults cause the controller does not function properly, as we observed for the thrusts 1 and 4 (Figure 11) and the control inputs 2, 3 and 4 (Figure 10), there is a time instant when its becomes negative and this is impossible, because it would mean that the thrust force is 

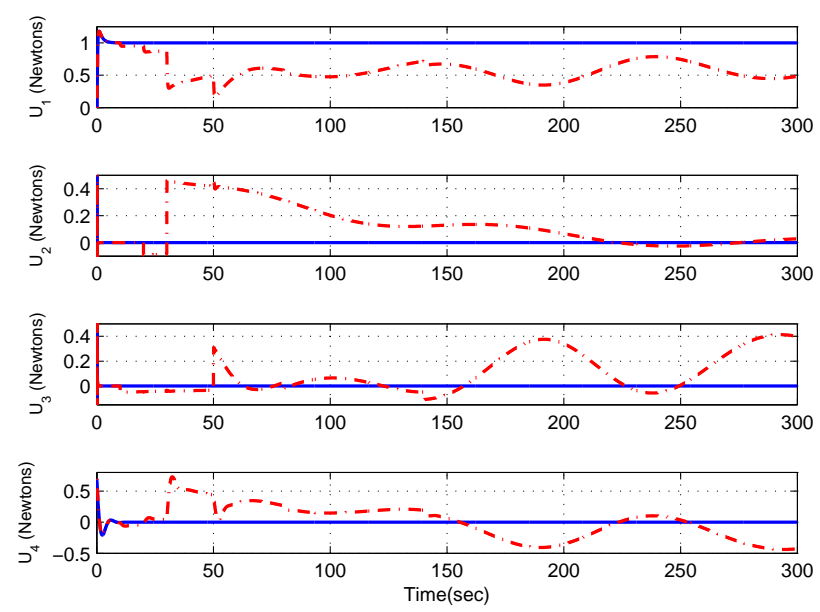

Fig. 10. Comparison for the inputs generated by the control strategy for the case without faults (blue line) and with faults (red dash-dot line)
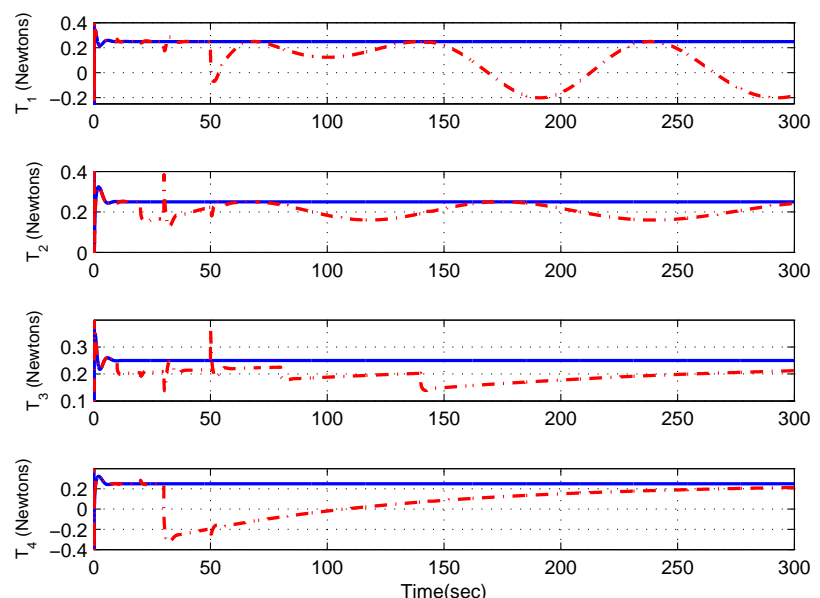

Fig. 11. Comparison for the corresponding thrust inputs for the case without faults (blue line) and with faults (red dash-dot line)

opposite, i.e, when the thrust force becomes negative, the engine does not have the ability to change the direction of rotation, and therefore stops completely (turned off) and in this case we are not dealing with a fault, we would have a failure. 


\section{Conclusion and Future Work}

This work deals with the problem of fault detection and diagnosis task for a Quad-rotor mini air vehicle (MAV) using the differential algebra approach. This approach consider the unknown faults like an augmented state of the system, the strategy is proposes a bank of observers in order to estimate the fault dynamics, in this case we are only use the available measurements and known inputs. A polynomial observer was proposed to deal with the fault estimation problem for the case of multiple faults. This approach detects and identifies multiple faults of relative small magnitudes. In this work the FDD task for a system stabilized in the closed-loop using a control strategy is presented.

The second part of this paper concentrates on the study of the controllability of the system with a failure. For that purpose, we have presented an evaluation function depending on system states. By means of this evaluation function, we can develop a control methodology which compensates the effect of the failure under certain considerations. That will be the subject of the second part of the paper.

\section{References}

1. G. Flores, J. Escareno, R. Lozano, and S. Salazar, "Quad-tilting rotor convertible mav: Modeling and real-time hover flight control," Journal of Intelligent and Robotic Systems, vol. 65, no. 1-4, pp. 457-471, 2012.

2. S. Bouabdallah and R. Siegwart, "Backstepping and sliding-mode techniques applied to an indoor micro quadrotor," in In Proceedings of IEEE Int. Conf. on Robotics and Automation, Barcelona, Spain, Apr. 2005, pp. 2247-2252.

3. G. Flores and R. Lozano, "Lyapunov-based controller using singular perturbation theory: An application on a mini-uav," in Proc. IEEE American Control Conference (ACC'2013), Washington, DC, Jun. 2013, p. to appear.

4. S. Bertrand, T. Hamel, and H. Piet-Lahanier, "Stability analysis of an uav controller using singular perturbation theory," in Proceedings of the 17th World Congress The International Federation of Automatic Control, Seoul, Korea, Jul. 2008, pp. 5706-5711.

5. G. Flores, L. Garcia, G. Sanahuja, and R. Lozano, "Pid switching control for a highway estimation and tracking applied on a convertible mini-uav," in Proc. 51st IEEE Conference on Decision and Control (CDC'2012), Maui, HI, USA, Dec. 2012, to be published.

6. H. Khebbache, "Robust control algorithm considering the actuator faults for attitude tracking of an uav quadrotor aircraft," International Journal of Control and Automation, vol. 5, no. 4, pp. 55-66, 2012.

7. A. Freddi, S. Longhi, and A. Monteriï£i, "A model-based fault diagnosis system for a mini-quadrotor," in 7th Workshop on Advanced Control and Diagnosis (ACD 2009), Zielona Gï£ira, Poland, Nov. 2009, pp. --

8. — - "Actuator fault detection system for a mini-quadrotor," in 2010 IEEE International Symposium on Industrial Electronics (ISIE), Bari, Italy, Jul. 2010, pp. $2055-2060$.

9. A. Freddi, A. Lanzon, and S. Longhi, "A feedback linearization approach to fault tolerance in quadrotor vehicles," in Proceedings of The 2011 IFAC World Congress, Milan, Italy, 2011. 
10. M. Ranjbaran and K. Khorasani, "Fault recovery of an under-actuated quadrotor aerial vehicle," in Decision and Control (CDC), 2010 49th IEEE Conference on, dec. 2010, pp. $4385-4392$.

11. M. Fliess, C. Join, and H. Sira-Ramirez, "Robust residual generation for linear fault diagnosis: an algebraic setting with examples," International Journal of Control, vol. 77, no. 14, pp. 1223-1242, 2004.

12. M. Fliess, H. Sira-Ramirez et al., "Control via state estimations of some nonlinear systems," in IFAC Symposium on Nonlinear Control Systtems (NOLCOS 2004), 2004.

13. J. J. Rincon-Pasaye, R. Martinez-Guerra, and A. Soria-Lopez, "Fault diagnosis in nonlinear systems: An application to a three-tank system," in American Control Conference, 2008. IEEE, 2008, pp. 2136-2141.

14. R. Martinez-Guerra and A. Luviano-Juarez, "Fault diagnosis of nonlinear systems using reduced-order observers and algebraic observers," in Decision and Control, 2006 45th IEEE Conference on. IEEE, 2006, pp. 544-549.

15. C. D. Garcia-Beltran, A. Rodriguez-Palacios, G. V. Guerrero-Ramirez, F. M. SilvaCarranza, and F. Sorcia-Vazquez, "Fault diagnosis of an induction motor based on differential algebra reconstructors," in World Automation Congress (WAC), 2012, june 2012, pp. $1-6$.

16. H. Aguilar Sierra, R. Martinez-Guerra, and J. Mata-Machuca, "Fault diagnosis via a polynomial observer," in Electrical Engineering Computing Science and Automatic Control (CCE), 2011 8th International Conference on, oct. 2011, pp. 1 -6 .

17. J. Mata-Machuca, R. Martinez-Guerra, and R. Aguilar-López, "An exponential polynomial observer for synchronization of chaotic systems," Communications in Nonlinear Science and Numerical Simulation, vol. 15, no. 12, pp. 4114-4130, 2010.

18. J. C. Cruz-Victoria, R. Martínez-Guerra, and J. J. Rincón-Pasaye, "On nonlinear systems diagnosis using differential and algebraic methods," Journal of the Franklin Institute, vol. 345, no. 2, pp. 102-118, 2008.

19. H. K. Khalil, Nonlinear Systems. New York: Prentice Hall, 2002.

20. P. Kokotović, H. K. Khalil, and J. O'Reilly, Singular Perturbation Methods in Control: Analysis and Design. London: Academic Press: Siam, 1999.

21. L. G. Carrillo, G. Flores, G. Sanahuja, and R. Lozano, "Quad-rotor switching control: An application for the task of path following," in Proc. IEEE American Control Conference (ACC'2012), Montreall, Canada, Jun. 2012, pp. 4637-4642. 